\title{
Disruptores endocrinos utilizados en la industria textil-confección en España
}

\author{
Endocrine disruptors used in \\ textile industry in Spain
}

\section{Rafael Gadea}

Instituto Sindical de Trabajo, Ambiente y Salud (ISTAS). España.

\section{Laura Mundemurra}

Federación de Industrias Textil, Química y Afines de Comisiones Obreras (CCOO). España.

Tatiana Santos

Instituto Sindical de Trabajo, Ambiente y Salud (ISTAS). España.

\section{Ruth Jiménez}

Instituto Sindical de Trabajo, Ambiente y Salud (ISTAS). España.

\section{Ana M García}

Instituto Sindical de Trabajo, Ambiente y Salud (ISTAS). España.

Departamento de Medicina Preventiva y Salud Pública.

Universidad de Valencia, Valencia, España.

CIBER Epidemiología y Salud Pública, España.

Correspondencia:

Ana M García

Instituto Sindical de Trabajo, Ambiente y Salud (ISTAS)

Ramón Gordillo 7, $1^{a}$

46010 Valencia

Tel: 963911421

Fax: 963917264

Ana.M.Garcia@uv.es

Financiación: El presente estudio se enmarca dentro del proyecto

"La prevención a la exposición a disruptores endocrinos en la Industria del Textil

y de la Confección", realizado con una ayuda concedida por la Fundación para

la Prevención de Riesgos Laborales (referencia IS-002/2006).

Resumen

Introducción: Los disruptores endocrinos son sustancias químicas que pueden alterar el sistema hormonal. Estas sustancias se utilizan en distintos procesos de la industria del textil-confección.

Objetivos: Identificar las sustancias con efectos de disrupción endocrina utilizados en la industria del textil y la confección en España para prevenir la exposición de los trabajadores a estas sustancias.

Material y métodos: En el estudio participaron 65 empresas de siete comunidades autónomas, seleccionadas mediante acuerdo entre las organizaciones empresariales y sindicales del sector. Técnicos de salud laboral de las federaciones sindicales visitaron las empresas participantes y recogieron información sobre los productos químicos utilizados mediante observación de etiquetas y fichas de datos de seguridad y mediante entrevistas con técnicos de prevención, trabajadores designados, delegados de prevención y trabajadores utilizando cuestionarios estandarizados.

Resultados: Las empresas participantes cubren un amplio rango de actividades propias del sector, siendo la mayoría de ellas de tamaño medio (entre 51 y 250 trabajadores, n=39). Se identificaron diecisiete 
sustancias diferentes con efectos de disrupción endocrina utilizadas en distintos puestos de trabajo, incluyendo preparación de fibras y tejidos, lavado, tintado o acabado textil, entre otros.

Conclusiones: Serían necesarios estudios que permitieran cuantificar el nivel de exposición en los puestos identificados para priorizar las medidas preventivas necesarias.

Palabras clave (MeSH): Disruptores endocrinos; Industria textil; Salud laboral, Med Segur Trab 2009; 55 (214): 111-118

\section{Abstract}

Introduction: Endocrine disruptors are chemicals which can affect hormonal system in human beings. These substances are used in several processes in the textile industry.

Objectives: Identifying chemicals with endocrine disruption potential used in Spanish textile industry to promoting risk prevention in exposed workers.

Material and methods: The study includes 65 companies located in seven different Spanish regions and selected through management and trade union organizations agreement. Occupational health technicians from the local trade unions visited participating companies and gathered information about chemicals in use through observation of available labels and safety data sheets and through personal interviews with technicians, safety representatives and workers using standardized questionnaires.

Results: Participating companies cover a wide range of typical activities in the textile industry, most of them being medium sized (51-250 workers, $n=39)$. Seventeen different chemicals acting as endocrine disrupters were identified in a variety of jobs, including fibre and tissues elaboration, washing, dyeing and finishing, among other.

Conclusions: It would be needed to evaluate the level of exposure to endocrine disruptors in these tasks in order to prioritize necessary preventive actions.

Key words (MeSH): Endocrine disruptors; Textile industry; Occupational Health, Med Segur Trab 2009; 55 (214): 111-118 


\section{INTRODUCCIÓN}

Los disruptores endocrinos son sustancias químicas, exógenas al organismo animal o humano, que actuando como agonistas o antagonistas hormonales pueden alterar la homeostasis del sistema endocrino ${ }^{1}$.

En las dos últimas décadas se han observado y descrito numerosos fenómenos de disrupción endocrina en la vida silvestre, peces y ecosistemas. En estudios experimentales con animales de laboratorio también se han reproducido este tipo de alteraciones. Asimismo, en numerosos trabajos se ha relacionado la exposición a sustancias con actividad de disrupción endocrina en humanos con determinadas enfermedades y trastornos, tales como hipospadias, malformaciones en el aparato genital masculino, reducción en la producción de espermatozoides, o tumores de testículo y mama ${ }^{2,3}$. Estos datos han impulsado la investigación biomédica sobre disrupción endocrina en los últimos años, sobre todo en relación con ciertas sustancias químicas como bisfenilos policlorados, pesticidas organoclorados como el DDT o ftalatos ${ }^{4,5}$.

La preocupación suscitada por los efectos de la exposición a disruptores endocrinos ha llevado, especialmente en los Estados Unidos y la Unión Europea, a desarrollar programas de identificación de estas sustancias. Así por ejemplo, los trabajos realizados al amparo de una estrategia comunitaria específica ${ }^{6}$ han dado como resultado la identificación de algo más de 300 sustancias distintas con evidencia confirmada o potencial de alteración endocrina.

Más de la mitad de estas sustancias se comercializan en la Unión Europea en cantidades superiores a 1.000 t, según se desprende del ejercicio de comparación de la lista de disruptores endocrinos incluida en la base de datos RISCTOX ${ }^{7}$ con el listado de sustancias peligrosas de alto volumen de producción (HPV) que se comercializan en la Unión Europea ${ }^{8}$.

Los usos principales de estas sustancias son conocidos, pero no se dispone de listados exhaustivos de usos ni de puestos de trabajo con potencial riesgo de exposición a disruptores endocrinos ${ }^{9}$. Se sabe que en distintos procesos de la industria del textilconfección se utilizan sustancias con capacidad de disrupción endocrina en productos utilizados como plaguicidas, detergentes, surfactantes, plastificantes, colorantes, tintas, metales, aceites minerales o retardantes de llama ${ }^{10}$.

El presente estudio tiene su origen en un proyecto de la Federación de Industrias Textil-piel, Químicas y Afines de CCOO, la Federación de Industrias Afines de UGT y el Consejo Intertextil Español, financiado por la Fundación para la Prevención de Riesgos Laborales, con el objetivo de identificar las sustancias con efectos de disrupción endocrina que se usan en la industria del textil y la confección en España, así como sus condiciones de uso. La finalidad última de dicho proyecto es la de promover la prevención de la exposición de los trabajadores a estas sustancias. En este trabajo presentamos los principales resultados del estudio de campo realizado en 65 empresas del mencionado sector.

\section{MATERIAL Y MÉTODOS}

El trabajo de campo se ha realizado a lo largo del año 2007 sobre una muestra de 65 empresas del sector de textil y confección localizadas en Andalucía ( $n=4)$, Cantabria $(n=1)$, Castilla-León $(n=3)$, Castilla-la Mancha $(n=7)$, Cataluña $(n=19)$, Galicia $(n=12)$ y Comunidad Valenciana $(n=19)$. Las empresas fueron seleccionadas mediante acuerdo entre las organizaciones empresariales y sindicales del sector, incluyéndose para la selección criterios de representatividad en relación con el tamaño de empresa y la pertenencia a los diferentes subsectores productivos de la industria textil-confección.

Técnicos de salud laboral de las respectivas federaciones sindicales visitaron las empresas con el objetivo de obtener una relación de los productos químicos utilizados mediante la recogida de etiquetas y fichas de datos de seguridad. También se solicitaban a la empresa listados de los productos utilizados en los diferentes procesos productivos. 
Otros datos relativos a los productos utilizados y sus condiciones de uso se obtuvieron mediante cuestionarios administrados a técnicos de prevención o trabajadores designados en la empresa, delegados de prevención y trabajadores, con los que se contactaba durante las visitas realizadas a las empresas participantes. La información incluía datos de la empresa y de las personas entrevistadas (anonimizados a los efectos del presente análisis), de los usos de los productos químicos identificados, de las estrategias de formación e información desarrolladas por la empresa en relación con el uso de los productos en cuestión, de los riesgos para la salud percibidos por su utilización y de las medidas de prevención disponibles. Los cuestionarios combinaban opciones de respuesta cerradas y abiertas. Se puede solicitar una copia del cuestionario utilizado a los autores.

El análisis que se presenta a continuación es básicamente descriptivo, incluyendo las frecuencias absolutas y relativas de los productos identificados en relación con características y actividad de las empresas participantes y con los puestos de trabajo donde se ha registrado su uso.

\section{RESULTADOS}

En la Tabla I se presenta la distribución de las empresas incluidas en el estudio por comunidad autónoma, actividad principal y tamaño. En estas empresas se recogieron un total de 357 cuestionarios contestados por técnicos de prevención o trabajadores designados $(n=61)$, delegados de prevención $(n=77)$ y trabajadores $(n=219)$.

Tabla I. Localización, actividad y tamaño de las empresas incluidas en el estudio ( $n=65)$.

Proyecto "La prevención a la exposición a disruptores endocrinos en la industria del textil y de la confección", ISTAS 2007.

\begin{tabular}{|c|c|c|}
\hline Comunidad Autónoma & $\mathbf{n}$ & $\%$ \\
\hline 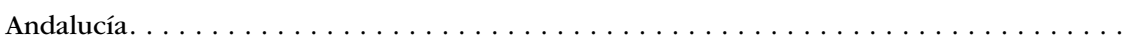 & 4 & 6 \\
\hline 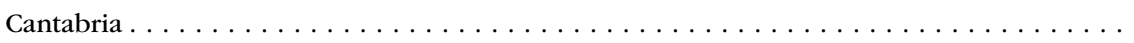 & 1 & 2 \\
\hline 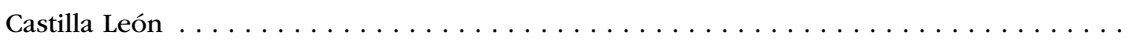 & 3 & 5 \\
\hline 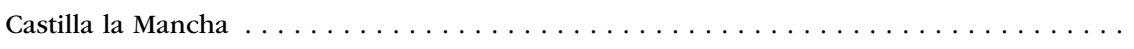 & 7 & 11 \\
\hline 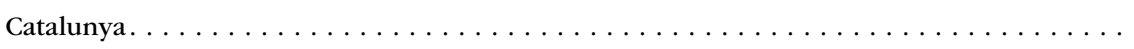 & 19 & 29 \\
\hline 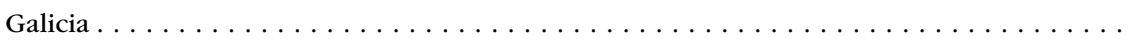 & 12 & 18 \\
\hline País Valencia & 19 & 29 \\
\hline Actividad (CNAE) & $\mathbf{n}$ & $\%$ \\
\hline Preparación e hilado de fibras textiles $(171) \ldots \ldots \ldots \ldots \ldots \ldots \ldots \ldots \ldots \ldots$ & 10 & 15 \\
\hline Fabricación de tejidos textiles $(172) \ldots \ldots \ldots \ldots \ldots \ldots \ldots \ldots \ldots \ldots \ldots$ & 7 & 11 \\
\hline 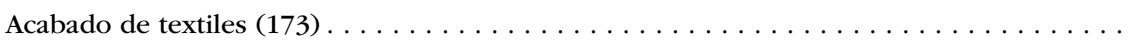 & 8 & 12 \\
\hline Fabricación de otros artículos confeccionados con textiles excepto prendas de vestir (174) & 6 & 9 \\
\hline Otras industrias textiles (175). . & 7 & 11 \\
\hline Fabricación de tejidos de punto $(176) \ldots \ldots \ldots \ldots \ldots \ldots \ldots \ldots \ldots \ldots \ldots$ & 5 & 8 \\
\hline Fabricación de artículos en tejidos de punto $(177) \ldots \ldots \ldots \ldots \ldots \ldots \ldots \ldots$ & 9 & 14 \\
\hline Confección de prendas de vestir y accesorios $(182) \ldots \ldots \ldots \ldots \ldots \ldots \ldots \ldots \ldots$ & 13 & 20 \\
\hline Tamaño de la empresa & $\mathbf{n}$ & $\%$ \\
\hline 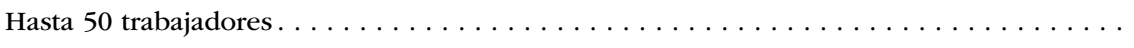 & 8 & 12 \\
\hline 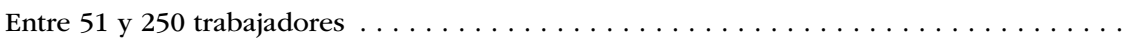 & 39 & 60 \\
\hline 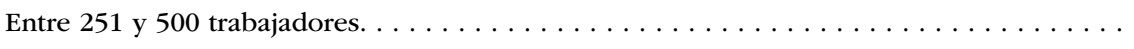 & 9 & 14 \\
\hline$>500$ trabajadores. . . . . . & 9 & 14 \\
\hline
\end{tabular}


Del total de situaciones analizadas, en los siguientes puestos de trabajo se refirió exposición a sustancias químicas: laboratorio, cocina de color, tintura, cuadrista, estampación, chamuscadota, devanadora, preparado y acabado de tejido y lavado, tejido, encolado, hilado, control de calidad, planchado, encargados, mantenimiento y otros sin especificar. Según los distintos informantes en las empresas, en estos puestos se utiliza una diversidad de familias de productos químicos, destacando por su frecuencia disolventes, tintas, colorantes, detergentes, lubricantes y colas. También se menciona el uso de emulsificantes, plastificantes, retardantes de llama, surfactantes y plaguicidas.

Tabla II. Número de productos y sustancias químicas identificados según actividad.

Proyecto "La prevención a la exposición a disruptores endocrinos en la Industria del Textil y de la Confección", ISTAS 2007.

\begin{tabular}{lcc}
\hline & $\begin{array}{c}\text { Preparados } \\
\text { químicos } \\
\text { n }\end{array}$ & $\begin{array}{c}\text { Sustancias } \\
\text { químicas } \\
\text { n }\end{array}$ \\
\hline Preparación e hilado de fibras textiles & 65 & 42 \\
Fabricación de tejidos textiles & 97 & 41 \\
Acabado de textiles & 694 & 393 \\
Otros textiles excepto prendas de vestir & 63 & 39 \\
Otras industrias textiles & 15 & 6 \\
Fabricación de tejidos de punto & 18 & 16 \\
Fabricación de artículos de tejidos de punto & 40 & 23 \\
Confección de prendas de vestir textiles y accesorios & 12 & 9
\end{tabular}

Tabla III. Sustancias con actividad como disruptores endocrinos identificadas y procesos en los que se ha registrado su uso. Proyecto "La prevención a la exposición a disruptores endocrinos en la Industria del Textil y de la Confección", ISTAS 2007.

\begin{tabular}{|c|c|c|}
\hline Sustancia & CAS & Proceso productivo \\
\hline Etilbenceno & $100-41-4$ & Tintado \\
\hline Disocianato de 4,4'-metilendifenilo & $101-68-8$ & Pretratamiento \\
\hline Acrilonitrilo & $107-13-1$ & Acabados \\
\hline Acetato de vinilo & $108-05-4$ & Acabados \\
\hline Anhídrido maléico & $108-31-6$ & Acabados \\
\hline 2-butoxietianol & $111-76-2$ & Mantenimiento, acabados \\
\hline Ftalato de bis (2-etilhexilo) & $117-81-7$ & Acabados \\
\hline Tetracloroetileno & $127-18-4$ & $\begin{array}{l}\text { Lavado, acabados, control de calidad, } \\
\text { mantenimiento, preparado de tejidos }\end{array}$ \\
\hline $\begin{array}{l}\text { Bisfenol diglicidil eter polímero } \\
\text { (resina epóxida) }\end{array}$ & $25068-38-6$ & Encolado \\
\hline Nonoxinol-9 & $26027-38-3$ & Acabados \\
\hline Ortoftalato de diisonilo & $28553-12-0$ & Acabados \\
\hline $\begin{array}{l}\text { Poli (oxi-1,2-etanodiil), alfa- } \\
\text { (isononilfenil)-omega-hidroxifosfato } \\
\text { (Un Nonilfenol etoxilato) }\end{array}$ & $37205-87-1$ & Acabados \\
\hline Permetrina & $52645-53-1$ & Acabados \\
\hline Etanol (anhidro) & $64-17-5$ & Acabados \\
\hline Diclorometano & $75-09-2$ & Mantenimiento, urdimbre, control de calidad \\
\hline Tricloroetileno & $79-01-6$ & Lavado, acabados, preparación de fibras, urdimbre \\
\hline Nonilfenol etoxilado & $9016-45-9$ & Acabados \\
\hline
\end{tabular}


La Tabla II muestra la distribución de preparados y sustancias químicas identificadas en los diferentes subsectores de la industria del textil y la confección, destacando por el volumen de productos referidos los subsectores de acabado de textiles, fabricación de tejidos, preparación e hilado de fibras textiles, y fabricación de otros textiles excepto prendas de vestir. Muchos de estos productos o sustancias aparecen identificados en diferentes procesos productivos o puestos de trabajo. El número de productos diferentes identificados se reduce a 500. De las diferentes sustancias identificadas, 288 disponen de número CAS.

En la Tabla III se presentan las sustancias identificadas como disruptores endocrinos. Estas sustancias se utilizan en los puestos de trabajo de preparación de fibras, pretratamiento, urdimbre, lavado, tintado, encolado, preparación de tejidos, acabados, control de calidad y mantenimiento. Algunas de ellas, como el tetracloroetileno, diclorometano o tricloroetileno, se utilizan en varios procesos diferentes. Otras sustancias han sido identificadas en un solo proceso, y la mayoría de ellas en el proceso de acabado textil.

\section{DISCUSIÓN}

En las 65 empresas del sector de textil y confección incluidas en el estudio se ha identificado el uso de 500 productos químicos y cerca de 300 sustancias diferentes. Estos productos se utilizan en diferentes puestos de trabajo de los tres principales procesos productivos del sector: ramo del agua (laboratorio, cocina de color, tintura, cuadrista, estampación, chamuscadota, devanadora, preparado y acabado de tejido y lavado), hiladura y tisaje (tejido, encolado e hilado), y algunos otros puestos comunes a los tres procesos (control de calidad, planchado, encargados, mantenimiento y otros sin especificar). Del total de sustancias identificadas, diecisiete son disruptores endocrinos. Estas sustancias se han identificado en relación con las actividades de preparación de fibras, pretratamiento, urdimbre, lavado, tintado, encolado, preparación de tejidos, acabados, control de calidad y mantenimiento. Según nuestro conocimiento, este es el primer estudio que permite identificar el uso de disruptores endocrinos en relación con actividades y puestos de trabajo en una muestra amplia de empresas del sector de textil y confección en diferentes comunidades autonómicas de España.

El desarrollo del trabajo de campo presenta limitaciones. Muy especialmente, las dificultades para conseguir por parte de las empresas los listados de las sustancias utilizadas, probablemente han permitido identificar tan sólo una parte de las sustancias químicas de uso en el sector del textil y la confección. Sin embargo, otras fuentes refieren que las sustancias empleadas en este sector podrían ser muchas más. Así por ejemplo, el Instituto de Investigación Ambiental de la Universidad de Dortmund (INFU) registra en su base de datos 1.970 sustancias de uso en el sector textil ${ }^{11}$. Otras fuentes ${ }^{10}$ elevan esta cifra a cerca de 10.000 sustancias, como puede observarse en la Tabla IV.

Tabla IV. Estimación del número de sustancias distintas utilizadas en la industria textil-confección en diferentes etapas del proceso productivo. Fuente: Enviro Tex $\mathrm{GMBH}^{10}$.

\begin{tabular}{|c|c|}
\hline Proceso & $\mathbf{n}$ \\
\hline 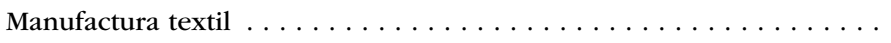 & 600 \\
\hline Apresto . . . . . . . . . & 400 \\
\hline 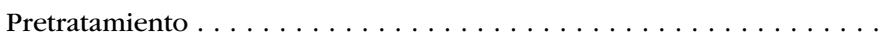 & 700 \\
\hline Tintura/impresión $\ldots \ldots \ldots \ldots \ldots \ldots \ldots \ldots \ldots \ldots \ldots \ldots \ldots \ldots$ & 2.600 \\
\hline 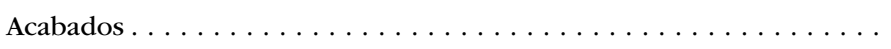 & 3.350 \\
\hline 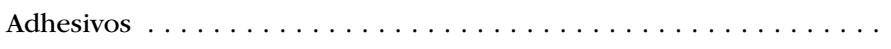 & 100 \\
\hline Recubrimientos . . & 400 \\
\hline Auxiliares varios. . . . . . . . . . . . . & 1.650 \\
\hline
\end{tabular}


Una explotación combinada de diferentes listados de sustancias utilizadas en la industria textil $^{12,13,14}$ con el listado de disruptores endocrinos de la base de datos RISCTOX ${ }^{7}$ permite caracterizar los grupos de sustancias que incluyen disruptores endocrinos, su uso y el proceso productivo en el que se utilizan o del que resultan como subproducto (como es el caso de los hidrocarburos aromáticos policíclicos). El número resultante de sustancias con propiedades de alteración endocrina es de 100, una cifra bastante superior al de sustancias con ese mismo efecto identificadas en el presente estudio. En la Tabla V se presenta una relación de las principales familias de sustancias con efectos de alteración endocrina potencialmente utilizadas en el sector textil según las distintas fuentes consultadas.

El desconocimiento de los usos de sustancias con propiedades de disrupción endocrina en las empresas y de la exposición de los trabajadores a las mismas impide la adopción de las medidas preventivas adecuadas. Serían necesarios estudios específicos que permitieran caracterizar cuantitativamente el nivel de exposición de los trabajadores afectados. Ello permitiría priorizar las medidas necesarias para la sustitución de los productos peligrosos por otros más seguros, o, en su caso, para controlar de forma adecuada la exposición de los trabajadores en las diferentes actividades donde se ha identificado la utilización de estas sustancias.

Tabla V. Características de las principales categorías de sustancias con acción como disruptores endocrinos utilizadas en la industria textil-confección (confección propia a parir de distintas fuentes, ver nota a pie de tabla).

\begin{tabular}{|c|c|c|c|}
\hline Sustancias & Propiedades & Proceso productivo & Fuente \\
\hline Colorantes & $\begin{array}{l}\text { Colorante; tinte; pigmento; auxiliar de } \\
\text { colorante }\end{array}$ & Tintado & (2) \\
\hline Fibras acrílicas & Ligante; aditivo & $\begin{array}{l}\text { Impresión; preparado de } \\
\text { fibras }\end{array}$ & (1) (3) \\
\hline $\begin{array}{l}\text { Fibras acrílicas; monómeros de } \\
\quad \text { vinilo }\end{array}$ & Polímero; ligante; aditivo & $\begin{array}{l}\text { Pre-tratamiento: seda; } \\
\text { preparado de fibras }\end{array}$ & (1) \\
\hline Difenil éteres polibromados & Pirorretardante & & (1) \\
\hline Esteres del ácido carboxílico & Auxiliar de colorante; portador & Tintado & (1) \\
\hline $\begin{array}{l}\text { Compuestos aromaticos clorados; } \\
\text { triclorobencenos }\end{array}$ & $\begin{array}{l}\text { Aglutinanteauxiliar de colorante; por- } \\
\text { tador }\end{array}$ & Tintado & (1) \\
\hline $\begin{array}{l}\text { Hidrocarburos aromáticos policí- } \\
\text { clicos }\end{array}$ & & & (1) \\
\hline Alquilfenol etoxilatos & $\begin{array}{l}\text { Surfactante; detergente; humectante; } \\
\text { nivelador; emulsionante; auxiliar de } \\
\text { colorante; agente de recubrimiento; } \\
\text { dispersante }\end{array}$ & & (1) \\
\hline Difenil éteres polibromados & Pirorretardante & & (1) \\
\hline Aceites de ésteres & Lubricante & & (1) \\
\hline Parafinas cloradas & Pirorretardante & & (1) \\
\hline Esteres del ácido carboxílico & Auxiliar de colorante; portador & Tintado & (1) \\
\hline Poliol ésteres & Lubricante; agente de recubrimiento & & (1) \\
\hline Fenoles & Portador; aglutinante & Tintado & (1) \\
\hline Hidrocarburos aromáticos & Portador & Tintado & (1) (3) \\
\hline $\begin{array}{l}\text { Bencenos halogenados; dicloroben- } \\
\text { cenos }\end{array}$ & Auxiliar de colorante; portador & Tintado & (1) \\
\hline
\end{tabular}

(1) Integrated Pollution Prevention and Control (IPPC). Reference Document on Best Available Techniques for the Textiles Industry. July 2003 [accedido 7 enero 2009]. Disponible en: http://eippcb.jrc.ec.europa.eu/pages/FActivities.htm

(2) Environmental Protection Agency. Survey of Chemical Substances in Consumer Products. Survey of chemical substances in textile colorants no. 58. 2005 [accedido 7 enero 2009]. Disponible en: http://www.mst.dk/Udgivelser/Publications/2005/06/87-76 14-677-4.htm

(3) OECD Series on Emission Scenario. Document on textile finishing industry. Documents \#7, 2004 [accedido 7 enero 2009]. Disponible en: http://www.olis.oecd.org/olis/2004doc.nsf/LinkTo/env-jm-mono(2004) 12. 


\section{AGRADECIMIENTOS}

El proyecto "La prevención a la exposición a disruptores endocrinos en la Industria del Textil y de la Confección" se ha realizado con una ayuda concedida por la Fundación para la Prevención de Riesgos Laborales (referencia IS-002/2006). Agradecemos también la colaboración del Consejo Intertextil Español, la Federación de Industrias Afines de la Unión General de Trabajadores (UGT), y la Federación de Industrias Textil, Química y Afines de Comisiones Obreras (CCOO).

\section{BIBLIOGRAFÍA}

1. Olea N, Fernández MF, Araque P, Olea-Serrano F. Perspectivas en disrupción endocrina. Gac Sanit. 2002;16(3):250-6.

2. International programme on chemical safety. Global assessment of the state-of-the-science of endocrine disruptors. Organización Mundial de la Salud 2002 [accedido 7 enero 2009]. Disponible en: http://www. ehponline.org/who/.

3. Committee on Toxicity, Ecotoxicity and the Environment. Opinion on human and wildlife health effects of endocrine disrupting chemicals, with emphasis on wildlife and on ecotoxicology test methods. DG XXIV 1999 [accedido 7 enero 2009]. Disponible en: http://ec.europa.eu/food/fs/sc/sct/out37_en.pdf.

4. Safe SH. Endocrine Disruptors and Human Health-Is There a Problem? An Update. Environ Health Perspect. 2000;108 (6): 487-93.

5. Wolff MS. Endocrine Disruptors. Challenges for environmental research in the 21st century. Ann NY Acad Sci. $2006 ; 1076$ : 228-38.

6. Communication from the Commission to the Council and the European Parliament. Community: Strategy for Endocrine Disrupters. Com (99)706 [accedido 7 enero 2009]. Disponible en: http://ec.europa.eu/environment/ docum/99706sm.htm

7. RISCTOX. Base de datos de sustancias químicas peligrosas. Instituto Sindical de Trabajo, Ambiente y Salud [accedido 7 enero 2009]. Disponible en: http://www.istas.net.

8. IUCLID CD-ROM. European Commission Joint Research Centre. Institute for Health and Consumer Protection. Public data on high volume chemicals. European Chemicals Bureau; 2000.

9. Institute for Environment and Health. Chemicals purported to be endocrine disrupters: a compilation of published lists [web report W20] [accedido 7 enero 2009]. Disponible en: www.silsoe.cranfield.ac.uk/ieh/ pdf/W20.pdf

10. Enviro Tex GmbH. Impact of Reach on Textile Supply Chains. December 16, 2005. Annex 3 [accedido 7 enero 2009]. Disponible en: http://ec.europa.eu/enterprise/reach/docs/reach/text_final_report_051216_en.pdf

11. OEKOpro. Institute for Environmental Research. University of Dortmund [accedido 7 enero 2009]. Disponible en: http://www.oekopro.de/search.php?l=EN.

12. Integrated Pollution Prevention and Control (IPPC). Reference Document on Best Available Techniques for the Textiles Industry. July 2003 [accedido 7 enero 2009]. Disponible en: http://eippcb.jrc.ec.europa.eu/ pages/FActivities.htm

13. Environmental Protection Agency. Survey of Chemical Substances in Consumer Products. Survey of chemical substances in textile colorants no. 58. 2005 [accedido 7 enero 2009]. Disponible en: http://www.mst.dk/ Udgivelser/Publications/2005/06/87-7614-677-4.htm

14. OECD Series on Emission Scenario. Document on textile finishing industry. Documents \#7, 2004 [accedido 7 enero 2009]. Disponible en: http://www.olis.oecd.org/olis/2004doc.nsf/LinkTo/env-jm-mono(2004)12 